in vivo $34: 2233-2248(2020)$

doi:10.21873/invivo.12034

Review

\title{
Review of the Literature on Leiomyoma and Leiomyosarcoma of the Adrenal Gland: A Systematic Analysis of Case Reports
}

\author{
MARIA SAKELLARIOU ${ }^{1}$, DIONYSIOS DELLAPORTAS ${ }^{2}$, MELPOMENI PEPPA ${ }^{3}$, \\ DIMITRIOS SCHIZAS ${ }^{4}$, EMMANOUIL PIKOULIS ${ }^{1}$ and KONSTANTINOS NASTOS ${ }^{1}$ \\ ${ }^{I}$ Third Department of Surgery, School of Medicine, \\ National and Kapodistrian University of Athens, Attikon University Hospital, Athens, Greece; \\ ${ }^{2}$ Second Department of Surgery, School of Medicine, \\ National and Kapodistrian University of Athens, Aretaieion University Hospital, Athens, Greece; \\ ${ }^{3}$ Department of Endocrinology, School of Medicine, \\ National and Kapodistrian University of Athens, Attikon University Hospital, Athens, Greece; \\ ${ }^{4}$ First Department of Surgery, School of Medicine, \\ National and Kapodistrian University of Athens, Laikon University Hospital, Athens, Greece
}

\begin{abstract}
Background/Aim: To date few cases of smooth muscle-derived tumors of the adrenal gland have been reported and their treatment remains a medical challenge. The aim of this manuscript was to systematically review the literature and present the tumor characteristics and their management in order to provide a standardized approach to their diagnosis and management. Materials and Methods: We searched five databases (PubMed, Scopus, Elsevier, ResearchGate, Google scholar) for relevant articles published until March 2020. Results: Twenty-two cases of adrenal leiomyoma, four cases of adrenal smooth muscle tumor and forty-five cases of adrenal leiomyosarcoma were included. Conclusion: We present the demographic, clinical, radiological, pathological and oncological characteristics and prognosis of tumors of the adrenal gland arising from smooth muscle cells, as well as describe the common clinical investigations and therapeutic modalities that have been reported as part of their management.
\end{abstract}

Adrenal tumors can be detected either incidentally or after investigation of signs and symptoms that they evoke. Generally,

This article is freely accessible online.

Correspondence to: Maria Sakellariou, MD, Third Department of Surgery, School of Medicine, National and Kapodistrian University of Athens, Attikon University Hospital, Rimini 1, Haidari, 12462, Athens, Greece. Tel: +30 6948404126, e-mail: marisakellariou@gmail.com

Key Words: Adrenal gland, neoplasia, suprarenal, leiomyoma, leiomyosarcoma, smooth muscle tumor, adrenalectomy, review. they can be histologically categorized as primary tumors of the adrenal cortex or medulla, metastases from other primary malignancies and other tumors of the adrenal gland which originate from connective tissue cells. Adrenal cortical tumors are comprised by (asymmetric) adrenal cortical hyperplasia, adenoma, myelolipoma, adrenocortical carcinoma, oncocytic adrenocortical neoplasms; medullary tumors are comprised by pheochromocytoma, ganglioneuroma, neuroblastoma, ganglioneuroblastoma and teratoma. Connective tissue origin tumors are rarer and are comprised mainly by haemangioma and lymphangioma with a few cases of leiomyoma, lipoma, hemangiosarcoma, lymphoma, leiomyosarcoma and liposarcoma, as reported in the literature. Patients with primary malignancies in lung, breast, kidney, colon, oesophagus, pancreas, liver, stomach or with melanoma can present with adrenal metastases spread from these tumors, which can commonly be found bilaterally (1).

Leiomyomas (LM) and leiomyosarcomas (LMS) are mesenchymal tumors that derive from smooth muscle cells. Their benign - leiomyoma or malignant - leiomyosarcoma characterization depends on nuclear atypia and/or pleomorphism, mitotic activity and tumor cell necrosis (2). Leiomyomas and leiomyosarcomas of the adrenal gland comprise a very small percentage of the gland's pathology with very little data available in the literature. The aim of this manuscript was to systematically review the literature and present demographic, clinical, radiological, pathological and oncological characteristics and prognosis of tumors of the adrenal gland arising from smooth muscle cells, as well as describe the common clinical investigations and therapeutic modalities that have been reported as part of their management. 


\section{Materials and Methods}

Literature search: We searched the internet medical database (PubMed, Scopus, Elsevier, ResearchGate, Google scholar) using the keywords -adrenal, leiomyosarcoma- and -adrenal, leiomyomaextending our search in the articles that were included in the references, as well as the articles that appeared in the suggestions as related articles until March $22^{\text {nd }} 2020$. We excluded from our review articles that were written in non-English language, as well as those that we could not retrieve in full-text form, or those that solemnly mentioned the tumors and did not include their characteristics. Our systematic search resulted in finding 45 cases of adrenal leiomyosarcoma (Figure 1), forty-three published as journal articles and one as a letter to the editor; two cases were described in the same article (3). Regarding non-malignant tumors our search concluded in 22 cases of adrenal leiomyoma and 6 cases of adrenal smooth muscle tumor (SMT) (Figure 2), all having been published as journal articles; two of them were reported in the same article (4).

Statistics. Size of tumors was calculated as maximum size of surgical specimen or maximum size shown at imaging studies if not resected or if histological examination size was not mentioned, bilateral tumors were counted separately. Image size was calculated as maximum size shown at imaging studies, bilateral tumors were included separately. Specimen size was measured as maximum size of surgical specimen.

Demographic data, tumor characteristics, known risk factors, imaging and pathology characteristics, extent of disease, and treatment strategies as well as progression and survival outcomes were collected. Patient survival and progression of disease outcomes were computed based on the last reported follow up in each publication. SPSS for Windows 22.0 was used for all statistical analyses.

\section{Results}

Leiomyoma. There have been described 28 cases of adrenal leiomyoma - adrenal smooth muscle tumor (SMT) in the English literature.

Age, gender, side, size. The median age at diagnosis is 34.5 years (average $=34.11$ years, minimum $=2$ years, maximum $=72$ years), with 20 cases being presented in females and the rest 8 in males. Eleven of these cases were presented as a mass in the left side, 8 in the right side and 9 had bilateral presentation, with median size upon diagnosis being $8 \mathrm{~cm}$ (average $=8.28 \mathrm{~cm}$, minimum $=3 \mathrm{~cm}$, maximum $=21 \mathrm{~cm}$ ) - out of 33 tumors (19 unilateral and 7 bilateral, 2 bilateral tumors provided no measurement) (4).

Symptoms, diagnosis, other locations. A total of $46.43 \%$ of cases presented with abdominal or flank pain/discomfort (517). A total of $14.29 \%$ of cases presented with weight loss and/or anorexia $(7,13,16,17)$ and malaise $(7,13,15,18)$. 1 case out of 28 had fever at the time of diagnosis (7), and 1 presented with weight gain (19). The symptoms duration lasted from 2 weeks (12) to months and years (10).
Regarding hormonal secretion from the tumor, one case that presented with hypertension showed a slight elevation of epinephrine and norepinephrine in the left adrenal vein and the left renal vein after inferior vena cava (IVC) blood sampling (20). One patient showed elevated plasma normetanephrine values during workup (11). One patient "was hypokalemic, but biochemical test results were not consistent with an aldosterone-producing adenoma" (8).

One case that was initially presented with diarrhoea was diagnosed with synchronous multiple submucosal SMTs in the large intestine (21) and another case had leiomyomas also in the spleen and the epicardium (22). Two cases reported by Suankratay et al. that manifested neurological symptoms had spinal cord and brain epidural SMTs along with bilateral adrenal SMTs (4).

Risk factors. A total of $50 \%$ of the cases were found to be associated with immunodeficiency either acquired (4-8, 12, $21,23-25)$ or congenital $(15,18,26)$. Five of these cases were also tested positive for Epstein-Barr virus (EBV) with in situ hybridization in the surgical $(4,5,15,18)$ or autopsy (24) specimen.

Two cases from the non-immunodeficiency associated leiomyoma group were also found positive for EBV in the surgical specimen $(11,14)$. In one human immunodeficiency virus (HIV)-negative case the surgical specimen was tested for EBV and was found negative (27). Five patients were investigated for EBV infection through IgG antibodies, two immunodeficient and one immunocompetent were found positive $(4,15,27)$ and two patients were found negative, one of each group $(8,17)$.

Comorbidities. One HIV-positive case that died due to sepsis was diagnosed with Non-Hodgkin's lymphoma of the brain in the autopsy findings (24), one case was associated with bilateral testicular microlithiasis and empty sella turcica (22), one case had non-insulin dependent diabetes melitus (28) and one patient had Hashimoto's thyroiditis (27). One patient had a history of hepatitis B infection (13), one had a synchronous ovarian mature cystic teratoma (29) and one patient with congenital immunodeficiency developed a large B-cell EBVpositive lymphoma 5 years after the adrenal leiomyoma was treated (18).

Imaging characteristics. A total of 23 out of 28 cases commented on the size of the tumor. The median size at diagnosis was $6 \mathrm{~cm}$ (average $=7.13 \mathrm{~cm}$, minimum $=2.1 \mathrm{~cm}$, maximum $=21 \mathrm{~cm})(5-17,19-23,25,27-30)$. A total of 26 cases commented on the imaging characteristics of the tumor. From these 26 cases $65.38 \%$ presented as a mass with high-density margins (enhanced capsule) with low-density areas in the center of the mass, suggestive of necrosis in Computed Tomography (CT) and/or with high-signal 


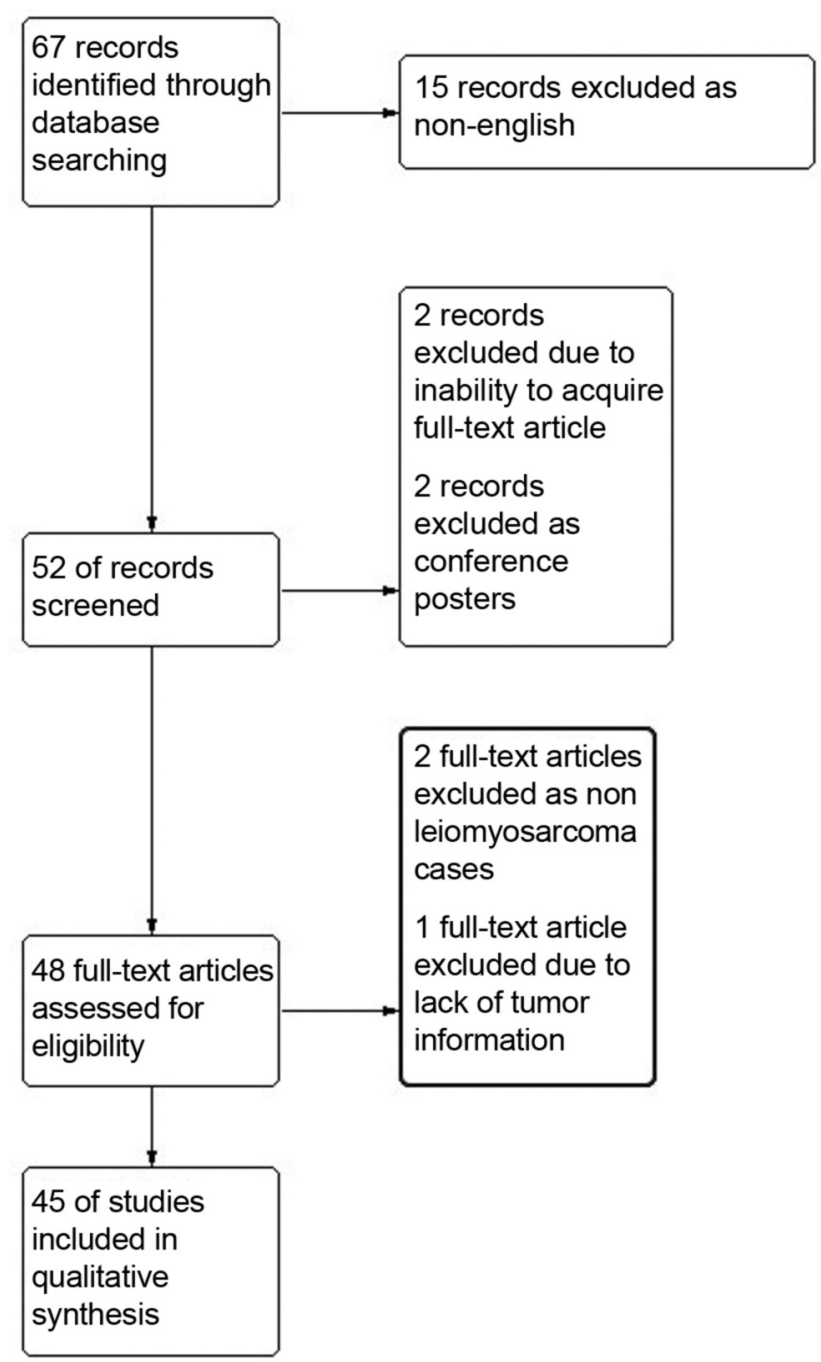

Figure 1. Adrenal leiomyosarcoma study flow diagram.

intensity center in T2-weighted (T2W) images in Magnetic Resonance Imaging (MRI) (5, 7-9, 11, 13, 15, 17, 18, 20-23, $25,26,28,30), 30.77 \%$ presented as a well-circumscribed mass $(7,11,12,20,21,23,26,28), 15.38 \%$ of the cases showed a heterogeneous mass with hypo-attenuated foci or calcifications $(9,10,15,16)$.

A total of $14.29 \%$ of the cases had a follow-up image study 4 months -3 years after diagnosis that showed increase of the mass $(7,8,21,27)$. Two cases showed possible IVC extension of the tumor, one of which was confirmed after an inferior venacavagram (25) and the other was found intraoperatively to be compressing the IVC without infiltrating it (13). One case had Hounsfield units (HU) values suggestive of malignancy (17) and one case showed an IVC defect suggestive of thrombus (15).

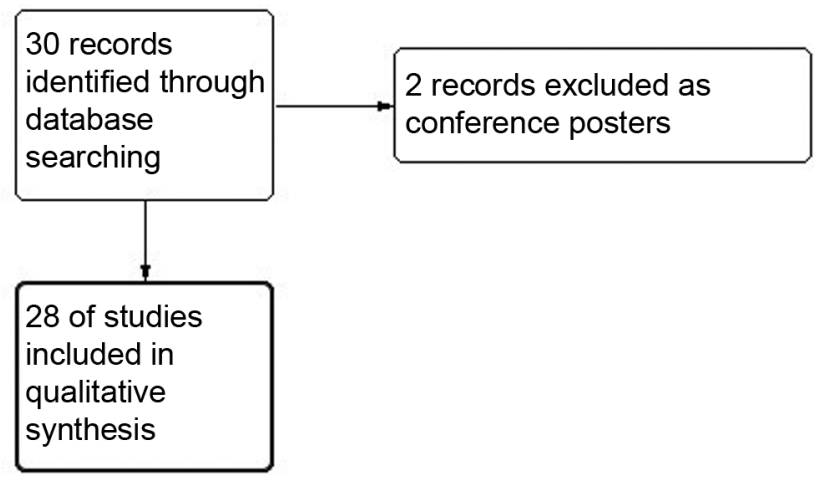

Figure 2. Adrenal leiomyoma study flow diagram.

Two cases presented as fluorodeoxyglucose (FDG) avid masses in Positron Emission Tomography (PET-CT) $(10,11)$, one case as negative (30), one case presented as metaiodobenzylguanidine (MIBG) scan negative (14) and one case showed faint uptake in MIBG scan (11).

Management. A total of $39.29 \%$ of the cases underwent an open unilateral adrenalectomy $(5-7,12,13,16,17,19,23$, $26,27)$. A total of $14.29 \%$ of the cases underwent bilateral open adrenalectomy $(11,14,18,25), 2$ cases had en bloc resection of the tumor with surrounding structures $(9,30), 2$ patients underwent laparoscopic adrenalectomy $(8,29)$, one patient had bilateral laparoscopic adrenalectomy (10), one case underwent hand-assisted laparoscopic adrenalectomy (28), one patient underwent staged tumor mass resection (15), one case with bilateral involvement had two operations, the first for the excision of one adrenal and the second for the excision of the contralateral adrenal mass as well as resection of splenic and epicardial masses (22), one patient underwent a second exploration 28 days after the first operation for the excision of a residual adrenal tumor (11). Suankratay et al. described two cases of bilateral adrenal SMTs with synchronous neural SMT one of which had partial tumor removal followed by radiotherapy (RT) and the second had partial spinal tumor removal, adrenal tumor biopsy and postoperative RT (4). One case did not undergo surgical excision (21) and one case was diagnosed postmortem (24).

Histological characteristics. Five cases underwent preoperative biopsy of the tumor, with three of them being non-diagnostic $(4,9,12)$ and the rest showing cells with characteristics of spindle cell neoplasm $(21,29)$.

The median surgical specimen's size measured was $9 \mathrm{~cm}$ (average $=9.17 \mathrm{~cm}$, minimum $=5 \mathrm{~cm}$, maximum $=18 \mathrm{~cm}$ ) out of 14 unilateral $(5,8,9,13,16,17,19,20,23,26-30)$ and 5 bilateral adrenalectomies $(10,11,14,18,22)$ and one 
autopsy specimen (24) that provided size and weighted in median $91 \mathrm{~g} \quad$ (average $=200.78 \mathrm{~g}, \quad$ minimum $=50 \mathrm{~g}$, maximum $=600 \mathrm{~g})$ out of 8 unilateral $(5,8,13,16,17,19$, $20,30)$ and 2 bilateral $(14,22)$ that provided weight.

From the 25 resected tumors and one autopsy specimen, 21 provided macroscopic characteristics of the tumor. A total of $71.43 \%$ of specimens comprised of firm - solid whitish/tanwhite/cream-colored mass $(5,8,10,13,14,17-20,22,23,26$, $27,29,30)$. A total of $38.10 \%$ of masses presented as encapsulated $(5,6,8,10,17,20,22,26)$, whereas $14.29 \%$ were non-encapsulated $(14,19,24)$ and one was surrounded by a pseudocapsule (18). A total of $33.33 \%$ of masses were described as well-defined or round $(6,11,16,23,24,28,30)$ and $23.81 \%$ were multilobulated $(10,11,14,18,23)$. Five cases presented a central area of hyalinization, haemorrhage, myxoid change or blot clot $(13,14,18,23,30)$, two had areas of calcification $(14,16)$ and one case presented as a unilocular cyst filled with blood clot (9). Two cases showed no areas of haemorrhage or necrosis $(5,17)$. Five cases did not provide such characteristics $(4,7,12,15,25)$ and two cases did not undergo resection of the adrenal mass $(4,21)$.

A total of 11 out of 14 cases that commented on the presence or absence of adrenal parenchyma in the surgical specimen found that adrenal parenchyma was present in the tissue $(7,8,10,16,18-20,22,24,26,30)$ and three stated that residual adrenal parenchyma could not be found or was totally occupied by the tumor $(9,13,27)$.

Microscopic examination of 25 cases that provided such characteristics $(2$ were not resected $(4,21)$ and 1 did not provide such description (7)) showed that $96 \%$ of tumors were comprised of spindle cells (4-6, 9-12, 14-20, 22-28, 30) $60 \%$ were arranged in intersecting fascicles $(4,9-11,14,16-$ $18,20,22,24,26-29)$. A total of $52 \%$ of specimens revealed no mitotic activity $(5,10,14,16,17,19,20,24-27,30)$, whereas $16 \%$ revealed low mitotic activity (1/10 high power field $(\mathrm{HPF}))(4,12,18,23)$. In one case with bilateral adrenalectomy, the examination of the left adrenal mass presented 5 mitosis/50 HPF and "was felt to be of indeterminate malignant potential" (25) and two cases revealed extension - infiltration of the IVC $(18,25) .36 \%$ of the tumors presented areas of hyalinized or myxoid connective tissue $(6,9-11,14,17,18,23,30), 24 \%$ showed no necrosis $(5,14,24,27,29,30), 20 \%$ showed areas of calcification $(9-11,14,16)$ with one of them presenting new bone formation (16) and $12 \%$ revealed areas of necrosis (9, $11,18)$. One case revealed numerous veins in the center of the tumor (8), three tumors had lymphocytic infiltration (15, $18,27)$ and one of them presented "a hemangiopericytomalike pattern" of the endothelial cell lining (15).

Of the 26 resected tumors, 20 provided immunohistochemical characteristics. Among them $75 \%$ were positive for smooth muscle actin (SMA) $(4,6,8-11,14-16,18,23,25,27$, $28,30), 30 \%$ were positive for desmin $(5,11,14,15,23,26)$ and another $15 \%$ had focally positive desmin staining $(6,18$, $22)$. A total of $25 \%$ of the tumors were positive for muscle specific actin (MSA) $(5,12,18,22,24)$ and for vimentin $(15$, $22,24,27,28)$. One case was found positive for S-100 (26), and another one for calponin and caldesmon staining (14). A total of $45 \%$ of the tumors were found negative for S-100 (4$6,10,18,22-24,30), 40 \%$ were found negative for CD34 (4, $6,16,18,23,27,28,30), 25 \%$ were negative for CD117 ( ckit) $(5,16,23,28,30), 20 \%$ were negative for cytokeratin staining (keratin/PAN-CK/CK6) $(6,18,23,26), 15 \%$ were negative for CD246 (ALK-1) $(16,18,23)$. Two cases were found negative for desmin staining $(27,30)$, for factor VIII $(24,26)$, for HMB45 $(4,14)$ and for EMA $(C D 227)(4,30)$. One case was found negative for myoglobin, myosin and alpha-1-antithrypsin staining (26), one for myogenin (23), one case was negative for $\beta$-catenin and for bcl-2 staining (30), one case was negative for estrogen receptor (ER), progesterone receptor (PR) and CD10 (16), one for CD31 and for LANA (HHV8) staining (6), one case was found negative for CD99 (4) and one case was found negative for PAX-5, CD2, CD3, CD138, CD20, CD4, CD8, CD30, CD21, CD56, CD5, CD68/PGM-1, Tbet and FOXP3 staining (18). One tumor was tested for Ki-67 marker and was found to be expressed in less than $5 \%$ of the tumor cells (18).

Diagnosis. The diagnosis based on pathology examination of the tumors was that of adrenal leiomyoma in $64.29 \%$ of the cases $(5-9,12,13,16,17,19,20,23,24,26-30)$, two of them were also confirmed through electron micrographs $(20$, 26 ), and that of bilateral adrenal leiomyomas in $10.71 \%$ of the cases $(10,11,14)$. One case was diagnosed with bilateral adrenal SMT, with the left tumor being characterized as of indeterminate malignant potential (25), one case was defined as bilateral benign or low-malignant EBV-SMT (18) and one case as bilateral EBV-associated SMT (15). One case with synchronous multiple submucosal SMTs in the large intestine was diagnosed as smooth-muscle tumor of the adrenal gland with minimal nuclear atypia through biopsy examination (21) and another case was diagnosed as multiple leiomyomas of the adrenals, spleen and epicardium (22). One case was diagnosed as EBV-associated SMT occupying both the adrenals along with the epidural of spinal cord at T4, T8 and L1 levels (4) and another case was diagnosed as EBVassociated SMT of the epidura of brain and spinal cord, vocal cord, orbit, and adrenal glands (4).

Follow-up. The longest follow-up period was 5 years in a patient who developed multifocal diffuse large B-cell lymphoma 5 years after bilateral adrenalectomy (18). Two cases documented no recurrence 26 months postoperatively $(9,15)$. One case showed no recurrence 1.5 years postoperatively under cortisone, Sa-fludrocortisone and testosterone therapy after bilateral adrenalectomy (22). Two 
Table I. Recent leiomyoma literature 2015-2020.

\begin{tabular}{|c|c|c|c|c|c|c|c|c|c|}
\hline Author & $\begin{array}{c}\text { Publication } \\
\text { year }\end{array}$ & Age & Gender & $\begin{array}{l}\text { Immuno- } \\
\text { deficiency }\end{array}$ & $\begin{array}{l}\text { Size } \\
(\mathrm{cm})\end{array}$ & EBV & $\begin{array}{l}\text { Surgical } \\
\text { resection }\end{array}$ & $\begin{array}{l}\text { Weight } \\
(\mathrm{g})\end{array}$ & Follow-up \\
\hline Meher et al. & 2015 & 42 & $\mathrm{~F}$ & $\mathrm{~N}$ & $12.2 \times 10.3 \times 8$ & $\mathrm{~N}$ & Left adrenalectomy & 91 & $\begin{array}{l}\text { NR } 6 \text { months } \\
\text { postop }\end{array}$ \\
\hline Corti et al. & 2016 & 40 & M & Y & $4.3 \times 5.7$ & ND & Left adrenalectomy & NA & NA \\
\hline Huei et al. & 2017 & 46 & M & Y & $7 \times 7.9 \times 8.8$ & ND & Right adrenalectomy & NA & $\begin{array}{l}\text { NR } 1 \text { year } \\
\text { postop }\end{array}$ \\
\hline Bastakoti et al. & 2019 & 60 & $\mathrm{~F}$ & $\mathrm{~N}$ & $5 \times 6$ & ND & $\begin{array}{c}\text { Right salpingo- } \\
\text { oopherectomy } \\
\text { followed by laparoscopic } \\
\text { left adrenalectomy }\end{array}$ & NA & NA \\
\hline Pramod et al. & 2020 & 34 & $\mathrm{~F}$ & ND & 14 & ND & Right adrenalectomy & 600 & NA \\
\hline
\end{tabular}

M: Male; F: female; Y: yes; N: no; ND: not defined; NA: not available; NR: no recurrence; postop: postoperatively; EBV: Epstein-Barr virus.

cases showed no recurrence 1 year postoperatively $(11,23)$. Autopsy of one patient that died due to sepsis 10 months postoperatively revealed no recurrence of the adrenal tumor (25). Two cases showed no recurrence 6 months after surgery $(14,17)$. One case that did not undergo surgical treatment revealed $50 \%$ increase in the diameter of the mass in a 10 month follow-up CT examination (21). One case with multiple SMTs developed residual tumors along with metastases in the abdominal wall 4 months after partial removal of the tumor and postoperative RT, and was last examined 2 years after diagnosis (4). One case with multiple SMT that had undergone partial neural tumor removal and postoperative RT was lastly observed 10 months after surgery (4). One case that was diagnosed as adrenal leiomyoma post-mortem through autopsy was that of an HIV-positive patient who died after progressive neurologic deterioration and sepsis (24). No follow-up data were available in 15 out of 28 of cases $(53,57 \%)$.

Recent literature regarding adrenal leiomyoma cases published in the last 5-year period are summarized in Table I.

Leiomyosarcoma. There have been described 45 cases of adrenal leiomyosarcoma in the English literature.

Size, location, age, gender. The reviewed literature shows that there is no preference of adrenal leiomyosarcomas in sex or side. The prevalence of adrenal leiomyosarcoma in males is 22 (out of 45) and 23 in females and in respect to side adrenal LMS were found to be 20 (out of 45) in the left side, 23 in the right side and 2 were bilateral - one of those cases being the bilateral recurrence of an excised left adrenal leiomyosarcoma 5 years earlier (31). The median age at diagnosis was 60 years (average $=55.56$ years, minimum $=14$ years, maximum $=81$ years) and the median size upon diagnosis was $9 \mathrm{~cm}$ (average $=9.83 \mathrm{~cm}$, minimum $=0.8 \mathrm{~cm}$, maximum $=27 \mathrm{~cm}$ ) out of 43 tumors [37 unilateral and 2 bilateral tumors, 2 autopsy unilateral specimens, 4 cases did not describe tumor size (32-35)].

Symptoms, diagnosis. A total of $71.11 \%$ of the cases presented with abdominal or flank pain/discomfort $(32,33$, $35-64) .13 .33 \%$ of the cases presented with weight loss and/or anorexia, $(34,39,47,51,56,59)$. A total of 3 cases out of 45 presented as an enlarging abdomen mass $(32,65$, 66), 4 presented with dyspnoea or chest discomfort (32-34, $67), 2$ cases had fever at the time of diagnosis $(33,56)$ and nausea $(50,68)$. Five cases out of 45 presented with leg swelling $(32,49,59,67,69) 1$ with cold feet sensation (35) and 2 cases had skin varices at the time of diagnosis $(67,69)$. One case presented with weight gain (32), 1 had back pain related with bone metastases (34), 1 had urinary track symptoms (70) and 3 were asymptomatic (71-73). 1 case was diagnosed during follow-up for previously resected adrenal leiomyosarcoma (31) and two cases were diagnosed postmortem due to HIV-related infections (3). The symptoms duration lasted from days or sudden onset (33) to several months and years (58).

Regarding hormonal secretion from the tumor, one case presented with markedly high levels of serum neuron specific enolase (NSE) which were normalised after excision (41). One case had elevated levels of NSE, IL-2 receptor, thymidine kinase activity and adrenocorticotropic hormone (ACTH) (36). One patient showed elevated serum CA125 values during workup (67).

Risk factors. Four cases of adrenal leiomyosarcoma out of 45 $(8.89 \%)$ where found to be associated with acquired immunodeficiency syndrome (AIDS) $(3,31,57)$, two of which corresponded to autopsy cases (3). From these four, 3 cases were associated with EBV infection, as found through in situ hybridization (EBER) in the surgical (57) or autopsy specimen 
(3), with the remaining case not having been tested for EBV (31). Two cases described by Boman et al. that presented positive EBER hybridization in the autopsy specimen, were found negative for EBV latent membrane protein 1 (LMP1) in the tumor cells (3). From the remaining literature 6 cases were tested negative for HIV infection $(44,49,58,61,66,71)$. From these, one case was also tested for EBV in situ hybridization of the surgical specimen and was found negative (71) and another case was found negative for EBV LMP1 in the tumor cells (56). None of the LMS patients was tested for plasma EBV antibodies.

Comorbidities. One HIV-positive case that died due to cytomegalovirus (CMV) infection and neurological decline was diagnosed with Kaposi's sarcoma (3), one case had a history of an abdominal gunshot wound 4 years prior to LMS diagnosis, treated by partial hepatectomy and cholecystectomy (59), another case was associated with numerous heterogeneous intrauterine fibroids (50). One case had a known celiac disease and a history of laparotomies due to intestinal obstruction (37), one patient was a hepatitis B virus (HBV) carrier (68) and one case had a history of mediastinal seminoma at the age of 10 , treated with RT to the chest and testes (38).

Imaging characteristics. A total of 43 out of 45 cases commented on the imaging characteristics of the tumors and 33 out of 45 commented also on the size of the tumor. The median size at diagnosis was $7.8 \mathrm{~cm}$ (average $=8.54 \mathrm{~cm}$, minimum $=3 \mathrm{~cm}$, maximum $=25 \mathrm{~cm})(31,36-38,40-42,44,46-$ $49,51-56,58-64,66-73)$.

Of the 43 cases that described the imaging characteristics of the tumor, $58.14 \%$ described the mass as heterogeneous $(31,32,36-38,40-42,45,46,48,50-53,57,58,61,62,64$, $66,67,72,73), 30.23 \%$ as well-defined $(31,32,40,46,47$, $52,58,61,63,64,66,68,71), 18.6 \%$ commented on the mass as having image characteristics of necrosis $(31,32,40$, $46,49,52,63,64)$, in 2 cases the mass was described as having irregular borders $(48,49)$ and in 1 as lobulated (57).

A total of $23.26 \%$ of the cases appeared extending to the IVC through tumor thrombus $(32,33,35,37,44,53,54,59$, $67,69), 13.95 \%$ showed infiltration of adjacent organs (kidney, liver, pancreas) $(34,35,48,53,65,70)$ and $16.28 \%$ appeared to have distant or lymph node metastases (pulmonary, bone, liver) $(34,38,51,53,63,65,73)$.

Seven cases were depicted as avid masses in PET-CT (34, $38,41,51,53,54,62), 5$ cases were described as negative in MIBG scintigraphy $(41-44,64)$ and 2 in aldosterone scintigraphy $(41,42)$. One case was tested through 67 gallium scintigraphy and was found positive (36).

Biopsy. In 9 cases a preoperative form of adrenal biopsy was performed $(32,34,38,45,49,51,57,59,69)$. Two cases were subjected to percutaneous fine needle aspiration (FNA) $(45,57), 6$ cases to core biopsy and one to both techniques (51). From these cases, 5 patients proceeded with surgical excision, one of them was deemed inoperable during exploratory laparotomy (59), and the remaining 4 were not qualified as surgical candidates due to either local extension or metastatic disease $(32,34,38,51)$. Regarding diagnosis through biopsy specimen, 5 specimens revealed the presence of a spindle cell neoplasm, with (57) or without smooth muscle differentiation $(43,55,47,49)$ and in 4 cases the diagnosis of a malignant spindle cell neoplasm was rendered $(30,32,36,67)$.

Lujan et al. reported the diagnosis of metastatic pulmonary carcinoma to the adrenal gland on a needle biopsy specimen and noted that the following "preoperative chemotherapy reduced only the pulmonary mass but not the adrenal mass, which continued to enlarge" (63).

Management. Thirty-eight cases were submitted to surgical resection, while 4 were deemed inoperable $(32,34,38,51)$, one was subjected to exploratory laparotomy but the tumor was found unresectable due to fixation to the right lobe of the liver (59), and two were found post-mortem in autopsy findings (3). Four patients were submitted to laparoscopic adrenalectomy $(46,55,58,72)$, one in bilateral laparoscopic adrenalectomy (31) and two were converted from laparoscopic to open procedure $(62,70)$.

In patients who were subjected to open procedures, kidney resection en bloc with the tumor was performed in 12 patients $(31.58 \%)(33,35,40,41,43-45,50,56,65,69,71)$, liver segment was removed in 3 patients $(48,53,65)$ and part of the pancreas along with the spleen was removed in 1 case (61). In a patient that underwent laparoscopic excision the tumor was resected en bloc with the infiltrated liver capsule (72). One patient underwent en bloc resection of infiltrated diaphragm (43) and another underwent resection of the tumor along with part of the posterior wall of the stomach (71). Tumor thrombus was removed in 6 patients $(33,35,44$, 67-69), infiltrated IVC wall was removed and reconstructed in 6 patients $(37,41,53,54,64,69)$. Cardiopulmonary bypass was necessary in 3 cases $(33,35,67)$.

Two cases underwent further operations for metastases (42) and local recurrence (71). One case that was deemed inoperable underwent thoracic surgery for metastases (38) and three cases underwent autopsy examination $(3,59)$. One patient received preoperative chemotherapy (65), 5 patients received combination chemotherapy with radiotherapy postoperatively $(39,44,45,50,73), 5$ patients received adjuvant chemotherapy $(36,49,54,61,71), 2$ patients received radiotherapy postoperatively $(35,64)$ and one patient received combination chemotherapy RT and radiofrequency ablation (RFA) postoperatively (42). One patient received combination chemotherapy and RT only 
(51), 2 cases underwent chemotherapy only $(32,38)$ and one patient received RT only (34). In total, 15 patients received chemotherapy and 10 radiotherapy. Most commonly, therapy was doxorubicin-based $(46.67 \%)(38,39,45,51,54,61,71)$ and gemcitabine-based $(13.33 \%)(49,50)$. One patient was treated with both regimens sequentially (73). One patient was treated with doxorubicin-based chemotherapy and then with cisplatin (42) and one other with receptor tyrosine kinase (RTK) and mechanistic target of rapamycin (mTOR) inhibitors (36). The patient treated with neoadjuvant chemotherapy received paclitaxel (65). Two cases did not provide information on chemotherapy regimens $(32,44)$.

Histological characteristics. Immunohistochemical data were available in 44 cases, 37 cases through surgical $(31,33,36$, $37,39-50,52-58,60-73), 3$ cases through autopsy $(3,59)$ specimen examination, while 4 cases of unresected tumors provided information through biopsy specimen examination $(32,34,38,51)$. In one case no information was provided regarding histological characteristics of the tumor (35).

The median surgical specimen's size measured was 10.5 $\mathrm{cm}$ (average $=11.22 \mathrm{~cm}$, minimum $=0.8 \mathrm{~cm}$, maximum $=27$ $\mathrm{cm})$ out of 27 unilateral $(37,39,40,43-45,47,49,50,52$, $54-58,60-62,64-67,69-73)$ and 3 autopsy specimens $(3,59)$ that provided size and weighted in median $482 \mathrm{~g}$ (average $=692 \mathrm{~g}$, minimum $=47 \mathrm{~g}$, maximum $=2400 \mathrm{~g}$ ) out of 10 unilateral tumors that provided weight $(37,39,40,47,50$, $52,56,70,72,73), 3$ cases that provided the weight of the surgical specimen containing en bloc the resected kidney were not included (43-45).

From the 27 surgical pathology and one autopsy report (59) that commented on the presence of normal adrenal tissue, 19 reported that adrenal tissue was present (39-41, 44, $45,47,50,53,56-58,61,62,64,66-68,71,73)$ and 9 did not find any normal adrenal cells within the specimen (33, $37,43,52,59,65,69,72)$.

From the 33 cases that provided macroscopic characteristics of the tumors $63.64 \%$ described a greyish - white-tan coloured mass $(3,33,37,40,41,43-45,52-54,57-60,62,66-71)$, $39.39 \%$ appeared lobulated/multinodular $(37,39,43,47,56$, $57,62,63,65-67,69,73), 27.27 \%$ were well-defined/roundish tumors $(3,39,45,56-58,60,64) .66 .67 \%$ of the tumors had areas of cystic degeneration/haemorrhage/necrosis or of myxoid change $(33,37,39-41,43-45,49,50,52-54,57,58$, $62,64,67,70-73) .27 .27 \%$ of the tumors were surrounded by capsule $(47,50,56,61,63,65-67,71)$ one was surrounded by a pseudo-capsule (64) and two were not encapsulated $(3,72)$. Three cases described extra-adrenal extension of the tumor $(39,45,50)$, two showed IVC invasion from the tumor (37, $54)$ and three revealed the presence of tumor thrombus in the adrenal vein $(60,62,68)$.

Microscopic characteristics were available for 44 tumors; 37 surgical, 4 biopsy and 3 autopsy specimens. A total of
95.45\% described a tumor being comprised of spindle cells (3, $31-34,36-47,49-64,66-73), 45.45 \%$ of tumors had cells arranged in interlacing fascicles $(3,33,38-41,45,50,55-58$, $60,62,64,66-68,71) .56 .82 \%$ of the tumor cells presented nuclear atypia or pleomorphism $(36,37,39,40,43-45,47,49$ $55,59,62,64-68,71-73), 45.45 \%$ had areas of necrosis (37$39,43,45,47,49-52,56,57,59,64,65,67,70,71,73)$, and $72.73 \%$ of the tumor cells showed marked mitotic activity (3, $33,37-41,44,45,47-50,52,54-57,59-67,70-73)$. From them 23 cases described the mitotic figures per HPF and $65.22 \%$ were found to have less than 10 mitotic figures/10HPF $(3,38$, $49,54,55,57,60,61,63,64,67,71-73)$ and $34.78 \%$ had more than 10/10HPF $(39,41,45,47,48,50,56,66) .18 .18 \%$ of the cases described the presence of giant tumor cells $(39$, $43,50,52,58,66,70,71)$, four described the presence of lymphocyte or plasma cell infiltration of the tumor $(3,31,62$, $70)$, two cases described eosinophilic infiltration $(47,63)$ and two cases commented on the presence of tumor cells in the adrenal vein wall $(68,73)$.

A total of 43 cases provided microscopic characteristics of the tumors; 36 resected tumors, 4 biopsy and 3 autopsy specimens. Out of 35 specimens that were tested for SMA $34(97.14 \%)$ were found positive $(3,31,33,34,36-38,41$ $45,47-49,53-62,64,66-71,73)$ and one negative (65). Out of 15 that were tested for MSA, $14(93.33 \%)$ were found positive $(3,31,32,34,39,45,50,55-57,60,64,72)$ and one negative (65). Of the 33 specimens that were tested for desmin $26(78.79 \%)$ were found positive (37-39, 44, 46-52, $54,56,58,60,61,63-69,71-73)$, three patchy positive (3, $32,55)$ and 4 were found negative $(3,31,43,62)$. Of the 22 that were tested for vimentin $20(90.91 \%)$ were found positive $(31,32,34,36,37,39,43-45,52,55,56,58,59$, 64-66, 70-72), one was weakly positive and one was found negative (3). Out of 33 specimens tested for S100, 31 $(93.93 \%)$ were found negative $(3,31,34,37-39,41,43,44$, $46-50,52,53,55-58,61,64-67,69-73)$, one was found positive (51) and one weakly positive (45). Out of 22 specimens tested for CD34, $19(86.36 \%)$ were found negative $(3,31,39,42,44,46,48,51,53,56,58,60,64-66$, $68,70,71)$, two were found positive $(55,61)$ and one was found patchy positive (38). Of the 13 specimens tested for CD117 (41, 46, 47, 55, 56, 58, 60, 61, 65, 66, 68, 70, 71) and of the 11 that were tested for HMB-45 $(39,46,47,62$, $64-68,71,72)$ all were found negative. Of the 7 tested for caldesmon $6(85.71 \%)$ were found positive $(48,55,61,64$, $69,70)$ and one negative (63). Both specimens tested for calponin were found positive $(50,65)$, and both negative for calretinin $(52,73)$, three that were tested for myoglobin were found negative $(46,65,71)$, three specimens tested for myogenin were found negative $(43,65,70)$ and one positive (49). Of the 19 specimens tested for cytokeratin 17 were found negative $(34,42,43,46,47,50,52,54-56,64-66,70$ 73 ), one was patchy positive (32) and one was found positive 
for CK Mak6 (51). Of 6 specimens tested for inhibin $(37,49$, $50,52,62,70)$ and of the 7 for chromogranin $(44,51,52$, $65,70,72,73)$ all were found negative. Of the five specimens tested for synaptophysin $(44,52,70,72,73)$ and for c-kit $(38,42,63,64,67)$ all were found negative. Of the three specimens tested for NSE two were found positive (41, 65 ) and one negative (44) and of the two tested for p53 one was found positive (39) and one moderately positive (43). Five tumors tested for $\operatorname{CD} 68(3,52,55,71)$, two tested for $\operatorname{ALK}(31,70)$, for $\operatorname{ER}(39,50)$, for bcl-2 $(54,58)$, for CD31 $(65,70)$, for Dog-1 $(58,61)$, for melanA $(50,72)$ and two for CMV, von Willebrand factor and BNH-9 (3) were found negative. One case was tested for factor VIII (57), one for CEA and EMA (65), one for RCC and CD10 (50), one for CD57 (41), one for HMWK (43), one for CD30 and Lambda and kappa light chain proteins (70), one for MITF (62) and were found negative. One tumor was tested for Smooth Muscle myosin heavy chain (37) and one for WT1 (51) and were found positive.

Seventeen tumors were tested for the Ki-67 proliferation marker. Of them two were expressing the marker in $<10 \%$ $(39,70)$, four in $20-30 \%(55,60,63,64)$, one moderately expressed the marker (43), three expressed it in $40-50 \%$ (53, $61,69)$, three in $>50-70 \%(49,54,58)$ and four in $\geq 75 \%(50$, $65,72,73)$.

Diagnosis. The diagnosis based on pathology examination of the tumors was in 29 cases $(64.45 \%)$ that of adrenal leiomyosarcoma $(3,32,33,35-37,40-42,45-47,49,51,52$, $54-61,63,64,66,69-71)$, in one case $(2.22 \%)$ of bilateral adrenal leiomyosarcoma (31), in two cases $(4.44 \%)$ that of leiomyosarcoma of the adrenal vein $(67,68)$, in 5 cases $(11.11 \%)$ of pleomorphic adrenal leiomyosarcoma $(39,43$, $50,65,72)$, in one $(2.22 \%)$ of dedifferentiated adrenal leiomyosarcoma (62) and in one (2.22\%) of multiple leiomyosarcomas (adrenal, pericardium, lymph nodes (LN)) (3). In 6 cases $(13.34 \%)$ the diagnosis was that of adrenal leiomyosarcoma with extra-adrenal extension, one with IVC extension (44), one with multiple metastases (34), one with liver metastasis (38) one with IVC extension and LN metastases (53), one with periadrenal soft tissue and liver invasion (48) and one with periadrenal extension (73). Of 17 cases that described the grade of leiomyosarcoma $6(35.29 \%)$ were defined as low grade $(3,39,57,66,70), 5(29.42 \%)$ as intermediate $(37,48,49,55,60)$ and $6(35.29 \%)$ as high grade $(32,35,43,47,69,71)$. Three cases commented in the differentiation of the tumors, two as well-differentiated (57, 73) and one as poorly-differentiated (33). Two cases commented in the staging of the tumors being pT2b $(60,69)$. Two cases commented on specific histological characteristics of the tumors, one as LMS with osteoclast-like giant cells (39) and one as LMS with eosinophilic infiltration (TATE: tumor-associated tissue eosinophilia) (47).
Extent of disease. Lymph node metastasis was confirmed in 2 cases at time of diagnosis, one in the aortocaval region (38) and one adjacent to IVC at the level of proximal portion of right renal vein (53). Distant metastases were reported in 4 cases at time of diagnosis, one patient with pulmonary (65) one with multiple bone and pancreatic metastases (34), two patients with simultaneous hepatic and pulmonary metastases $(38,51) .10$ cases report invasion of locoregional structures, eight of them report IVC tumor extension $(32,33,35,37,44,53,54,69)$, one of periadrenal soft tissue and liver (48) and one of the periadrenal fatty tissue (73).

Follow-up - Oncological outcomes. Follow-up data were available in 38 cases. In two cases the diagnosis was established post-mortem (3) and 5 cases did not provide follow-up information $(31,51,52,61,63)$. From the 38 cases that described the course of the patients, $18(47.38 \%)$ had no recurrence in a follow-up period from 1.5 months (43) to 3 years (55) (median $=12$ months, average $=13.14$ months) $(40,41,43,46,47,53,55-58,60,62,64,66-68,70$, 72). Six cases $(15.80 \%)$ reported development of local recurrence or metastases without a fatal outcome, in a timeto-recurrence period from 2 months (49) to 9 months (50) (median $=3$ months, average $=4.33$ months) and alive-withdisease period of 3 (48) to 31 months (73) (median=7.50 months, average $=10.17$ months $)(45,48-50,54,73)$. Two cases $(5.25 \%)$ reported the development of local recurrence 12 months (39) and 17 months (71) after surgery, then undergone a second operation along with chemotherapy and RT (39) and had a disease-free survival of 24 months (39) and 12 months (71) afterwards. 10 cases $(26.32 \%)$ reported the development of local recurrence/metastases and ultimately the death of the patients from a period shortly after surgery (65) to 16 months postoperatively (42), median time to recurrence $=1.5$ months, average $=2.88$ months, median time to death $=5$ months, average $=6.18$ months $(32$, $33,35-37,42,44,59,65,69)$. From the above mentioned, 6 cases $(60 \%)$ had a reported IVC invasion at diagnosis $(32$, $33,35,37,44,69)$. Two cases $(5.25 \%)$ with distant metastases at the time of diagnosis reported the death of the patient one month (34) and 9 months after diagnosis (38) respectively.

From the patients that developed distant metastases, seven developed liver metastases $(36,37,39,42,44,50$, $73)$, five pulmonary $(33,37,50,69,73)$, five bone metastases $(38,42,44,45,73)$, four $\mathrm{LN}$ metastases $(33,37$, $49,50)$, two pleural $(38,42)$, one metastases of the brain (34) and two cases reported metastases in the ipsilateral kidney and pancreas $(37,42)$.

Recent literature regarding adrenal leiomyosarcoma cases published in the last 5-year period are summarized in Table II. 


\section{Discussion}

Tumors of smooth muscle cell origin present very rarely in the adrenal gland. They take the form of either benign leiomyomas or malignant leiomyosarcomas. Adrenal leiomyomas have been described in less than 30 cases in the English literature with almost half of them being associated with HIV-positive patients. Adrenal leiomyosarcomas are retroperitoneal tumors in the suprarenal region with less than 50 cases described in the English literature. They are thought to derive from the adrenal vein and/or its branches.

Both of these tumors are very difficult to diagnose preoperatively as they are not frequently accounted in adrenal specimens and clinical suspicion is very low. As a result, they are diagnosed postoperatively after pathological examination of the surgical specimen, as they have no specific clinical or imaging characteristics nor specific biomarkers for their preoperative diagnosis and are usually discovered after they have reached a significant size due to compressive symptoms. It is due to their increased size and non-secreting profile that they are usually misdiagnosed as adrenocortical carcinomas.

Both adrenal leiomyomas and leiomyosarcomas are most usually depicted as large heterogeneous masses which occasionally have central necrosis. Leiomyosarcomas may often extend to the IVC. Benign tumors have rarely been reported to extent to the IVC, consequently, histologic evaluation of the two benign tumors that displayed IVC extension lead to their characterisation as having undetermined malignant potential $(18,25)$. The lack of specific imaging characteristics of these tumors makes them undistinguishable from other adrenal lesions, such as adrenal cortical carcinoma or pheochromocytoma, so the newlydiagnosed patient will usually undergo surgical excision. The first approach of the patient preoperatively is the evaluation of tumor secretion to confirm or exclude the diagnosis of a functional tumor (74). The dexamethasone suppression test and the plasma cortisol and ACTH levels can help to identify a cortisol-producing tumor. Aldosterone-producing adrenal tumors can be diagnosed by evaluating the levels of plasma aldosterone and dehydroepiandrosterone (DHEA)-producing tumors can be identified by the measurement of the plasma DHEA levels. Pheochromocytoma can be diagnosed by testing the levels of plasma and urinary metanephrines and catecholamines, accompanied by a MIBG scintigraphy (75).

Our analysis showed that a significant part of patients presenting with leiomyosarcoma have common predisposing factors associated with HIV and EBV infection. Although pathogenesis of the adrenal leiomyosarcoma remains unknown, there have been described 4 cases in the English literature where it occurs in patients with AIDS. Zetler et al. and Boman et al. investigated the tissue specimens from 3 cases for EBV genome, by EBV-encoded RNA in in-situ hybridization and they found the neoplastic cells strongly positive, whereas the adjacent non-neoplastic cells and negative controls showed no hybridization signals. Boman et al. also tested the tissue specimens by latent membrane protein immunocytochemistry (EBV LPM1) which were found negative in both specimens $(3,31,57)$. Leiomyomas are associated with immunodeficiency and EBV infections even more frequently. Out of 28 cases of adrenal leiomyoma - SMT 9 cases had HIV infection $(5-8,12,21,23-25)$ one had ataxia-telangiectasia (26), one had common variable immunodeficiency (CVID) (18) and one case had Natural Killer cell deficiency (15).

The imaging characteristics could not be specifically defined by our review, as there was great inconsistency in the imaging modality used between studies as well as the reported details of each modality. In most cases masses with increased size, infiltration of surrounding structures, tumor thrombus and heterogenicity in the tumor's density consistent with necrosis were present. However, an observation worth noting is that there were reports of lack of leiomyosarcoma metastases 18-FDG-PET/CT avidity while primary tumors were avid $(38,54)$.

We also found a discrepancy in the terminology of these tumors. Smooth muscle cell tumors of the adrenal gland are thought to derive from the adrenal vein or its branches, considering its smooth muscle differentiation. However, it is not clear if all tumors originate from the adrenal vein or other smaller intra adrenal vasculature. The reason is that when these tumors become symptomatic there is extensive growth within the adrenal gland making it impossible to clearly define the origin. Only 3 cases of leiomyosarcomas have been described in the English literature that a direct neoplastic growth within a vein is witnessed in the microscopic examination of the tumor $(62,68)$ and it is significant to note that in the case described by Shao et al., along with the case reported by Wang et al., the tumors have been reported as leiomyosarcomas of the adrenal vein and not of the adrenal gland. On the other hand, Wang et al. mention that "this finding was consistent with leiomyosarcoma, most likely arising from adrenal vein; however, on multiple sections, no residual vascular wall was detected. Primary adrenal leiomyosarcoma was considered unlikely due to clear-cut separation of the tumor from the morphologically unremarkable adrenal gland by a thin rim of fibrous tissue". Our team was the third to publish a case where the tumor clearly seems to grow within the wall of an adrenal vein (73). Consequently, the incidence of adrenal vein origin of the tumors cannot easily be defined as it cannot be clearly reported histologically and as a result all tumors should probably be considered as a single clinical entity.

Our literature review found histological variations in leiomyosarcomas. Smooth cell tumors of the adrenal gland are comprised by spindle cells, with eosinophilic cytoplasm and cigar-shaped blunt-ended nuclei, that are arranged in 


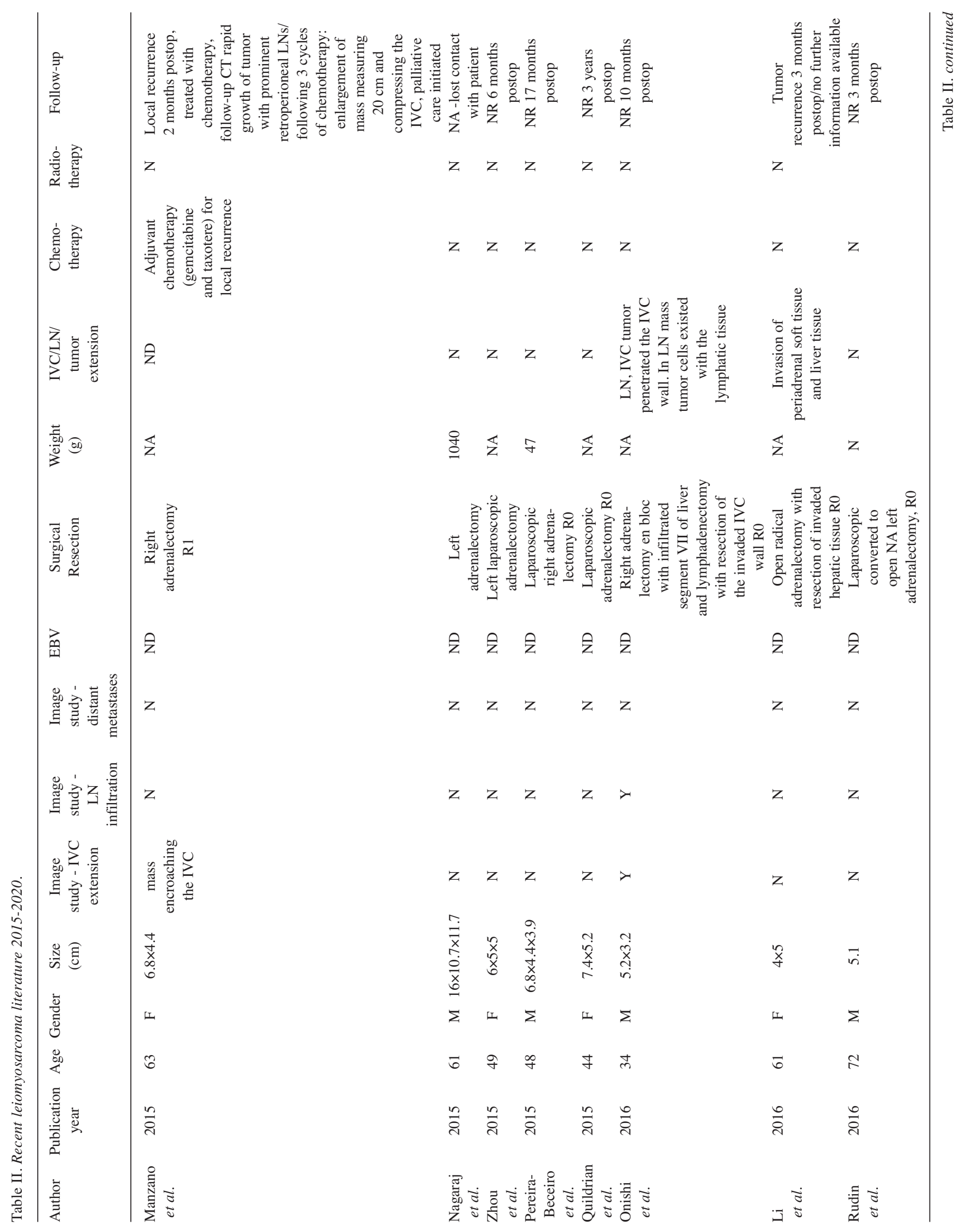




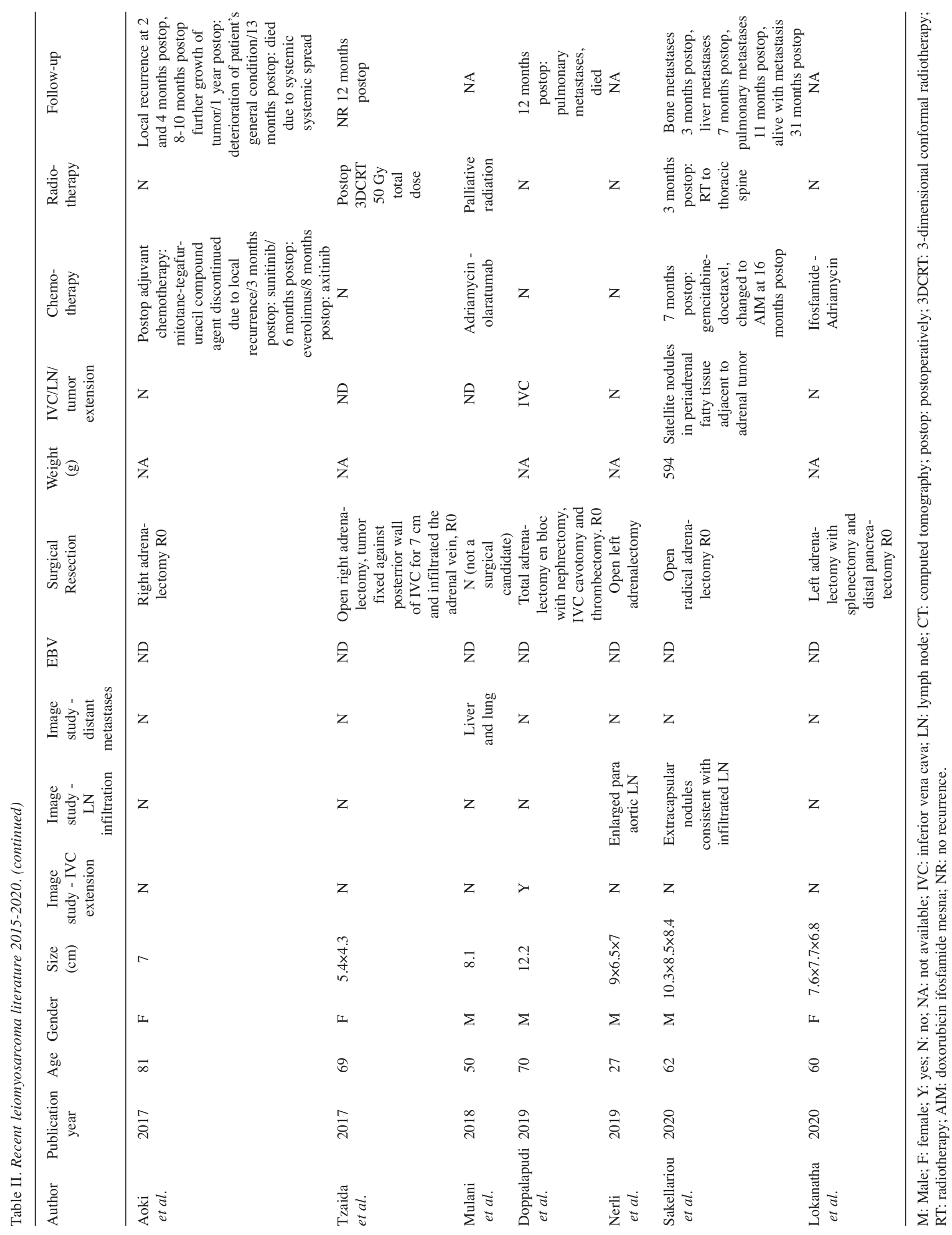


interlacing fascicles (2). Usually the mass is surrounded by a pseudo-capsule and residual adrenal tissue can be identified stretched in the periphery of the tumor. High-grade tumors often have areas of necrosis, haemorrhage or cystic degeneration. There have been described 5 cases of pleomorphic adrenal leiomyosarcoma, which is a morphologic variant of leiomyosarcoma that consists of pleomorphic areas mimicking storiform-pleomorphic malignant fibrous histiocytoma (MFH) in more than two thirds of the tumor and an ordinary leiomyosarcomatous fascicular area covering less than one third of the tumor $(39,43,50,65,72,76)$.

Lastly, a case of a primary adrenal dedifferentiated LMS where the mass showed two distinct components one being a low-grade leiomyosarcoma and the other a high-grade dedifferentiated element with focal and weak SMA reactivity, with the transition between the two being sharp has been described (62).

Although immunohistochemical staining pattern does not seem to be constant and the spectrum of markers tested differed significantly between cases, tumors were found to be positive for muscle-specific-actin (MSA/HHF35) in 14 of 15 reported of cases, alpha-smooth-muscle-actin ( $\alpha$ SMA) in in 34 of 35 reported of cases, vimentin in 20 of 22 reported cases and desmin in 26 of 33 reported cases. Tumors were found to have a high rate of mitosis as Ki-67 stain was $>20 \%$ in 14 of 17 reported cases. Due to its location in the retroperitoneal suprarenal region, the tumor must be immunohistochemically tested and differentiated from adrenocortical carcinoma (MelanA, synaptophysin, inhibin, calretinin, S100), neuroendocrine tumors (chromogranin, synaptophysin, S100, NSE), melanoma (MelanA, S100, HMB45), epithelial tumors (cytokeratins) $(77,78)$.

The gold standard of treatment for adrenal malignancy is surgical resection with negative margins and free of residual microscopic disease. Traditionally, preoperative FNA biopsy is not recommended, as there is a high risk of tumor seeding and diagnostic yield is low for primary adrenal malignancy (79). In our review we identified 9 cases where a biopsy of the adrenal tumor was performed, either by CT-guided FNA, or ultrasound (US)-guided FNA, by percutaneous thick needle biopsy, or by endoscopic ultrasound (EUS)-guided Core biopsy (32, 34, 38, 45, 49, 51, 57, 59, 65). Among these cases diagnostic yield was quite high clearly showing spindle cell neoplasia within the adrenal gland. This is in contrast to benign tumors where 3 from 5 cases of biopsy were found to be non-diagnostic $(4,9,12,21,29)$. As for the risk of local recurrence, Quildrian et al. reported 36 months recurrence free survival after complete surgical resection of the mass, which was the longest reported period (57). Regarding HIV-positive patients the longest recurrence free survival (RFS) was reported by Zetler et al., being 20 months after surgery (55). Others reported significantly smaller RFS, accompanied by distant metastases.
Leiomyomas were almost always removed surgically and the follow-up period was without recurrences, except in two cases reported by Suankratay et al. where multiple tumors thought to be benign showed metastatic spread and local recurrences after suboptimal surgical debulking and postoperative radiotherapy. (4). These two cases could potentially have been misdiagnosed as benign tumors. Regarding leiomyosarcomas our literature review showed that 5 patients were deemed inoperable and other treatment options were considered. From the 38 patients that proceeded with a surgical operation 5 had laparoscopic adrenalectomy $(31,47$, $55,58,72$ ) with maximum reported survival being 3 years (55) and 2 had laparoscopic converted-to-open adrenalectomy $(62,70)$ with maximum reported survival being 8 months (70). Although the kidney was removed in several cases due to suspicion of infiltration from imaging or intra-operative findings, only one case had microscopic confirmation of infiltration (42). The same was true for liver infiltration (53, $59,72)$, showing that preoperative evaluation of tumor infiltration is overestimated. We cannot recommend a specific approach based on our review of the literature, as there is certainly a selection bias in the surgical approach of patients, with larger tumors and tumors with suspicion of nearby organ infiltration being considered for open approach.

We found that tumor thrombus or IVC extension is present in nearly one fourth of leiomyosarcoma cases published in the English literature and an attempt to R0 resection (no residual tumor resection) should probably be performed. There have been described 11 cases where there was involvement of the IVC either by fixation of the tumor to the IVC wall or by extension of the tumor thrombus from the adrenal vein to the IVC. This mostly affected tumors of the right adrenal gland. Maximum survival with no evidence of disease in these patients was 12 months postoperatively reported by Tzaida et al., and 10 months after surgery reported by Wang et al., Goto et al. and Onishi et al., which is accomplished by $\mathrm{R} 0$ resection margins of the tumor, resection of invaded IVC wall and excision of tumor thrombus with graft replacement if needed $(32,33,35,37$, 41, 44, 53, 54, 64, 67, 69).

Regional LN metastasis was confirmed in only 2 cases at time of diagnosis showing that unlike other retroperitoneal sarcomas, these tumors may metastasize to the lymph nodes more often $(38,53)$. However, the value and the extent of routine lymphadenectomy remains unclear from our review.

Distant metastases at time of diagnosis were reported in 4 cases $(34,38,51,65)$ with all of them being either at the lungs, liver, pancreas or bones, but also in the brain later in the course of the disease (38). Lujan et al. was the only reported case where the primary tumor was removed despite having spread to metastases and the patient died shortly after surgery. Surgery with a curative intent and follow up was available in 34 patients. From 25 cases that reported yearly 
follow up, 1-year survival rate was calculated at $60 \%$, while 13 cases reported 24 months follow up in patients with a 2year survival rate of $15.3 \%$. There were two cases that reported 3-year survival and none of the cases reported 5year survival. In 17 cases there was no evidence of disease progression during the follow up period (median follow $\mathrm{up}=12$, range $=3-36$ months). Median time to disease progression was 9 months (range $=0.5-36$ months).

The use of chemotherapy and radiation therapy in leiomyosarcomas remains controversial and is usually limited for advanced disease or unresectable tumors. From the literature reviewed 7 patients were administered chemotherapy $(32,36,38,49,54,65,71), 4$ radiation therapy $(34,35,44,64)$ and 5 patients a combination of chemotherapy and radiation therapy $(39,42,45,50,51)$. From the patients receiving chemotherapy alone, four patients received chemotherapy as an adjuvant postoperative treatment due to local recurrence or metastases $(36,49,54$, 71 ), with longest survival being reported by Wei et al. with local recurrence reported at 17 months postoperatively, and no recurrence after a second operation and adjuvant chemotherapy after 29-month follow-up. Two patients received chemotherapy as the only oncological treatment due to advanced disease at diagnosis which rendered them inoperable $(32,38)$. The most commonly administered regimens were based on doxorubicin and gemcitabine.

Radiation therapy was administered only in two patients as a monotherapy as part of metastatic symptom control and another two were treated adjuvantly after surgery for locoregional control. As a result, safe conclusions could not be made for this treatment modality $(34,44,64)$.

Overall, from the reviewed literature, the longest survival was reported by Quildrian who described the case of a 44year-old woman who underwent laparoscopic right adrenalectomy for a $12 \mathrm{~cm}$ grade 2 FNCLCC (Fédération Nationale de Centres de Lutte Contre le Cancer classification) adrenal leiomyosarcoma and remained without evidence of local or distant recurrence three years after surgery. The same survival period was reported by Candanedo-Gonzales et al. although the patient underwent a second operation 12 months after the adrenalectomy for local recurrence and liver metastases as well as adjuvant chemotherapy and radiotherapy and was alive with no evidence of disease 24 months later (37). Second longest survival was reported by Sakellariou et al. being 31 months postoperatively with metastatic disease (71). Long term survival was not reported for any patients found in the present review of the literature.

\section{Conclusion}

Adrenal tumors derived from smooth muscle cells consist rare tumors of the suprarenal region that are usually diagnosed after they have reached a large size or after they have provoked symptoms due to applying pressure to adjacent structures. Their diagnosis in an advanced stage contributes to their aggressive behavior and the poor disease free and overall survival in cases of malignancy. Their radiological characteristics are not specific and due to similarities with adrenal malignancy they should be considered in the differential diagnosis of a suprarenal nonsecreting malignant tumor. Malignant tumors may well be one of the most aggressive tumors of the adrenal gland, although a publication bias is certainly associated with the poor outcomes reported in this systematic review. To this end, although data from case series and observational trials are still lacking, aggressive treatment should probably be adopted in the management of these tumors.

\section{Conflicts of Interest}

All Authors declare that there are no conflicts of interest regarding this study.

\section{Authors' Contributions}

EP and KN designed the study. MS and DD wrote the article. MS and MP collected the data. DS, EP and KN offered scientific advice. $\mathrm{DD}, \mathrm{MP}$ and DS revised the manuscript. $\mathrm{KN}$ was the supervisor.

\section{References}

1 Lam AK-y: Update on adrenal tumours in 2017 world health organization (who) of endocrine tumours. Endocr Pathol 28(3): 213-227, 2017. PMID: 28477311. DOI: 10.1007/s12022-0179484-5

2 Miettinen M: Smooth muscle tumors of soft tissue and nonuterine viscera: Biology and prognosis. Mod Pathol 27(1): S17S29, 2014. PMID: 24384850. DOI: 10.1038/modpathol.2013.178

3 Boman F, Gultekin H and Dickman PS: Latent epstein-barr virus infection demonstrated in low-grade leiomyosarcomas of adults with acquired immunodeficiency syndrome, but not in adjacent kaposi's lesion or smooth muscle tumors in immunocompetent patients. Arch Pathol Lab Med 121(8): 834-838, 1997. PMID: 9278611.

4 Suankratay C, Shuangshoti S, Mutirangura A, Prasanthai V, Lerdlum S, Shuangshoti S, Pintong J and Wilde H: Epstein-barr virus infection-associated smooth-muscle tumors in patients with aids. Clin Infect Dis 40(10): 1521-1528, 2005. PMID: 15844077. DOI: $10.1086 / 429830$

5 Alteer M, Ascott-Evans B and Conradie M: Leiomyoma: A rare cause of adrenal incidentaloma. JEMDSA 18(1): 71-74, 2013. DOI: $10.1080 / 22201009.2013 .10872308$

6 Corti M, Carolis L, Veliz L and Campitelli A: Adrenal leiomyoma: A rare tumor presented as an incidentaloma in a patient with Aids. Mathews J HIV AIDS 1(1): 006, 2016.

7 Dahan H, Beges C, Weiss L, Abitbol M, Ledreff O, Teman G, Belattar K and Rymer R: Leiomyoma of the adrenal gland in a patient with aids. Abdom Imaging 19(3): 259-261, 1994. PMID: 8019358. DOI: $10.1007 / \mathrm{BF} 00203522$ 
8 Gibbs KE, White A and Kaleya R: Laparoscopic management of an adrenal leiomyoma in an aids patient. A case report and review of the literature. JSLS 9(3): 345, 2005. PMID: 16121885.

9 Goldman RL and Brodey PA: Symptomatic leiomyoma of the adrenal. Clin Imaging 18(4): 277-278, 1994. PMID: 8000956. DOI: 10.1016/0899-7071(94)90008-6

10 Kumar S, Nanjappa B, Agrawal P and Pushkarna A: Large bilateral adrenal leiomyomas presenting as calcified adrenal masses: A rare case report. Korean J Urol 55(5): 363-366, 2014. PMID: 24868343. DOI: 10.4111/kju.2014.55.5.363

11 Parelkar SV, Sampat NP, Sanghvi BV, Joshi PB, Sahoo SK, Patel $\mathrm{JL}$ and Oak SN: Case report of bilateral adrenal leiomyoma with review of literature. Pediatr Surg Int 29(6): 655-658, 2013. PMID: 23354505. DOI: 10.1007/s00383-013-3264-1

12 Parola P, Petit N, Azzedine A, Dhiver C and Gastaut J-A: Symptomatic leiomyoma of the adrenal gland in a woman with aids. AIDS 10(3): 340, 1996. PMID: 8882677. DOI: 10.1097/ 00002030-199603000-00016

13 Pramod SV, Siregar S, Safriadi F, Hernowo BS and Firdaus GI: The largest adrenal leiomyoma: A case report and literature review. Urol Case Rep 29: 101106, 2020. PMID: 31908964. DOI: 10.1016/j.eucr.2019.101106

14 Sathe PA, Shah HU, Kothari KS, Ranganathan S and Kandalkar BM: Bilateral epstein-barr virus-associated adrenal leiomyomas in a child without an established immunodeficiency. Pediatr Devel Pathol 15(4): 329-332, 2012. PMID: 22483165. DOI: 10.2350/12-01-1135-CR.1

15 Shaw RK, Issekutz AC, Fraser R, Schmit P, Morash B, MonacoShawver L, Orange JS and Fernandez CV: Bilateral adrenal ebvassociated smooth muscle tumors in a child with a natural killer cell deficiency. Blood 119(17): 4009-4012, 2012. PMID: 22427204. DOI: 10.1182/blood-2011-10-385377

16 Monsefi N, Dehghani M, Nowshadi PA, Mirkharrazi SM, Karimi A and Dabiri S: Leiomyoma of the adrenal gland presenting as an incidentaloma. Arch Iran Med 14(6): 419-422, 2011. PMID: 22039848. DOI: 011146/AIM.0012

17 Meher D, Dutta D, Giri R and Kar M: Adrenal leiomyoma mimicking adrenal malignancy: Diagnostic challenges and review of literature. J Endocrinol Metab 5(5): 304-308, 2015. DOI: $10.14740 /$ jem $318 \mathrm{w}$

18 Petrilli G, Lorenzi L, Paracchini R, Ubiali A, Schumacher RF, Cabassa P and Facchetti F: Epstein-barr virus-associated adrenal smooth muscle tumors and disseminated diffuse large b-cell lymphoma in a child with common variable immunodeficiency: A case report and review of the literature. Int J Surg Pathol 22(8): 712721, 2014. PMID: 21454372. DOI: 10.1177/1066896911399901

19 Jacobs IA and Kagan SA: Adrenal leiomyoma: A case report and review of the literature. J Surg Oncol 69(2): 111-112, 1998. PMID: 9808515. DOI: 10.1002/(sici)1096-9098(199810)69: 2<111::aid-jso12>3.0.co;2-z

20 Nishida S, Tanimura A, Takasaki S, Nagaoka S, Fukueda M, Ikeda S, Matsuo K, Akao M and Tokunaga M: Surgically resected adrenal leiomyoma: Report of a case. Surg Today 25(5): 455-457, 1995. PMID: 7640478. DOI: 10.1007/BF00311827

21 Radin DR and Kiyabu M: Multiple smooth-muscle tumors of the colon and adrenal gland in an adult with aids. AJR Am J Roentgenol 159(3): 545-546, 1992. PMID: 1503021. DOI: 10.2214/ajr.159.3.1503021

22 Demirel Ş, Erk O, Akkaya V, Tunaci A, Tanakol R, Terzioğlu T, Demiryont M, Kadioğlu A and Şenkal Ö: Multiple vascular leiomyomas involving bilateral adrenal glands, spleen, and epicardium, associated with bilateral testicular microlithiasis and empty sella turcica. J Pediatr Surg 32(9): 1365-1367, 1997. PMID: 9314265. DOI: 10.1016/s0022-3468(97)90324-9

23 Huei TJ, Lip HTC, Rahman M and Arulanantham S: Large adrenal leiomyoma presented as adrenal incidentaloma in an aids patient: A rare entity. Med J Malaysia 72(1): 65-67, 2017. PMID: 28255146.

24 Jimenez-Heffernan JA, Hardisson D, Palacios J, Garcia-Viera M, Gamallo C and Nistal M: Adrenal gland leiomyoma in a child with acquired immunodeficiency syndrome. Pediatr Pathol Lab Med 15(6): 923-929, 1995. PMID: 8705202. DOI: 10.3109/ 15513819509027028

25 Rosenfeld D, Girgis WS and Underberg-Davis SJ: Bilateral smooth-muscle tumors of the adrenals in a child with aids. Pediatr Radiol 29(5): 376-378, 1999. PMID: 10382219. DOI: $10.1007 / \mathrm{s} 002470050611$

26 Mouchet F, Ninane J, Gosseye S, Verellen C, Bonnier C, Evrard P, Vermylen C, Scheiff J-M and Cornu G: Leiomyoma of the suprarenal gland in a child with ataxia-telangiectasia. Pediatr Hematol Oncol 8(3): 235-241, 1991. PMID: 1742182. DOI: $10.3109 / 08880019109033457$

27 Al-Masri AA, Aladily TN and Younes NA: Unusual association between adrenal leiomyoma and autoimmune disease. SMJ 31(2): 199-201, 2010. PMID: 20174739.

28 Chang TH, Lee YC, Liu CC, Huang CH and Wu WJ: Adrenal leiomyoma treated by hand-assisted laparoscopic adrenalectomy: A case report. Kaohsiung J Med Sci 22(11): 575-579, 2006. PMID: 17110347. DOI: 10.1016/S1607-551X(09)70355-9

29 Bastakoti S, Lamichhane N, Shrestha S and Dhungana I: Adrenal leiomyoma: A rare tumor presented as an incidentaloma in a women with ovarian mature cystic teratoma. Nepalese J Cancer 3(1): 54-56, 2019. DOI: 10.3126/njc.v3i1.25917

30 Lin J, Wasco MJ, Korobkin M, Doherty G and Giordano TJ: Leiomyoma of the adrenal gland presenting as a non-functioning adrenal incidentaloma: Case report and review of the literature. Endocr Pathol 18(4): 239-243, 2007. PMID: 18197480. DOI: 10.1007/s12022-008-9013-7

31 Linos D, Kiriakopoulos AC, Tsakayannis DE, Theodoridou M and Chrousos G: Laparoscopic excision of bilateral primary adrenal leiomyosarcomas in a 14-year-old girl with acquired immunodeficiency syndrome (aids). Surgery 136(5): 1098-1100, 2004. PMID: 15523410. DOI: 10.1016/j.surg.2003.07.007

32 Karaosmanoglu AD and Gee MS: Sonographic findings of an adrenal leiomyosarcoma. J Ultrasound Med 29(9): 1369-1373, 2010. PMID: 20733196. DOI: 10.7863/jum.2010.29.9.1369

33 Matsui Y, Fujikawa K, Oka H, Fukuzawa S and Takeuchi H: Adrenal leiomyosarcoma extending into the right atrium. Int J Urol 9(1): 54-56, 2002. PMID: 11972651. DOI: 10.1046/j.14422042.2002.00413.x

34 Van Laarhoven HW, Vinken M, Mus R, Flucke U, Oyen WJ and Van der Graaf WT: The diagnostic hurdle of an elderly male with bone pain: How 18f-fdg-pet led to diagnosis of a leiomyosarcoma of the adrenal gland. Anticancer Res 29(2): 469-472, 2009. PMID: 19331188.

35 Wong C, Von Oppell UO and Scott-Coombes D: Cold feet from adrenal leiomyosarcoma. J R Soc Med 98(9): 418-420, 2005. PMID: 16140856 . DOI: 10.1258/jrsm.98.9.418

36 Aoki C, Tanaka S, Kunihiro S, Sagara M and Aso Y: Primary adrenal leiomyosarcoma in an aged japanese woman: A rare case 
report. J Clin Case Rep 7(3): 935, 2017. DOI: 10.4172/21657920.1000935

37 Azzouni F, Schwaab T and Azabdaftari G: Primary adrenal leiomyosarcoma: Case report and review of literature.North Am J Med Sci 5(1): 58, 2012. DOI: 10.7156/v5i2p058

38 Bhalla A, Sandhu F and Sieber S: Primary adrenal leiomyosarcoma: A case report and review of the literature. Conn Med 78(7): 403-407, 2014. PMID: 25195305.

39 Candanedo-Gonzalez FA, Vela Chavez T and Cerbulo-Vazquez A: Pleomorphic leiomyosarcoma of the adrenal gland with osteoclast-like giant cells. Endocr Pathol 16(1): 75-81, 2005. PMID: 16000850. DOI: 10.1385/ep:16:1:075

40 Choi SH and Liu K: Leiomyosarcoma of the adrenal gland and its angiographic features: A case report. J Surg Oncol 16(2): 145148, 1981. PMID: 7464153. DOI: 10.1002/jso.2930160205

41 Goto J, Otsuka F, Kodera R, Miyoshi T, Kinomura M, Otani H, Mimura Y, Ogura T, Yanai H, Nasu Y and Makino H: A rare tumor in the adrenal region: Neuron-specific enolase (nse)producing leiomyosarcoma in an elderly hypertensive patient. Endocr J 55(1): 175-181, 2008. PMID: 18250540. DOI: 10.1507/endocrj.k07e-020

42 Hamada S, Ito K, Tobe M, Otsuki H, Hama Y, Kato Y, Sugiura Y, Kaji T, Asano $\mathrm{T}$ and Hayakawa M: Bilateral adrenal leiomyosarcoma treated with multiple local therapies. Int J Clin Oncol 14(4): 356-360, 2009. PMID: 19705248. DOI: 10.1007/ s10147-008-0844-5

43 Kanthan R, Senger JL and Kanthan S: Three uncommon adrenal incidentalomas: A 13-year surgical pathology review. World J Surg Oncol 10: 64, 2012. PMID: 22540324. DOI: 10.1186/14777819-10-64

44 Kato T, Kato T, Sakamoto S, Kobayashi T, Ikeda R, Nakamura $\mathrm{T}$, Akakura $\mathrm{K}$, Hikage $\mathrm{T}$ and Inoue $\mathrm{T}$ : Primary adrenal leiomyosarcoma with inferior vena cava thrombosis. Int J Clin Oncol 9(3): 189-192, 2004. PMID: 15221604. DOI: 10.1007/ s10147-004-0383-7

45 Lack EE, Graham CW, Azumi N, Bitterman P, Rusnock EJ, O'Brien W and Lynch JH: Primary leiomyosarcoma of adrenal gland. Case report with immunohistochemical and ultrastructural study. Am J Surg Pathol 15(9): 899-905, 1991. PMID: 1951846. DOI: 10.1097/00000478-199109000-00011

46 Lee CW, Tsang YM and Liu KL: Primary adrenal leiomyosarcoma. Abdom Imaging 31(1): 123-124, 2006. PMID: 16177871. DOI: $10.1007 / \mathrm{s} 00261-005-0343-3$

47 Lee S, Tanawit GD, Lopez RA, Zamuco JT, Cheng BG and Siozon MV: Primary leiomyosarcoma of adrenal gland with tissue eosinophilic infiltration. Korean J Pathol 48(6): 423425, 2014. PMID: 25588632. DOI: 10.4132/KoreanJPathol. 2014.48.6.423

48 Li C-C, Liu W-Y, Chang C-P, Kuo C-Y and Hwa Kuo J: Rare care report: Primary leiomyosarcoma of the adrenal gland and literature review. Urol Sci 27(2): s58, 2016. DOI: 10.1016/ j.urols.2016.05.212

49 Manzano AJ, Nose V, Fernandez-Castro GL and Ayala A: Recurrent primaty adrenal leiopyosarcoma: A complete literature review and presentation of a rare adrenal tumor. AACE Clin Case Rep 1(1): e8-e11, 2015. DOI: 10.4158/ep14197.cr

50 Mohanty SK, Balani JP and Parwani AV: Pleomorphic leiomyosarcoma of the adrenal gland: Case report and review of the literature. Urology 70(3): 591 e595-597, 2007. PMID: 17905130. DOI: 10.1016/j.urology.2007.07.029
51 Mulani SR, Stoner P, Schlachterman A, Ghayee HK, Lu L and Gupte A: First reported case of endoscopic ultrasound-guided core biopsy yielding diagnosis of primary adrenal leiomyosarcoma. Case Rep Gastrointest Med 2018: 8196051, 2018. PMID: 30402300. DOI: 10.1155/2018/8196051

52 Nagaraj V, Mustafa M, Amin E, Ali W, Naji Sarsam S and Darwish A: Primary adrenal leiomyosarcoma in an arab male: A rare case report with immunohistochemistry study. Case Rep Surg 2015: 702541, 2015. PMID: 25685588. DOI: 10.1155/ 2015/702541

53 Onishi T, Yanagihara Y, Kikugawa T, Miura N, Noda T, Kakuda T, Kitazawa R and Tanji N: Primary adrenal leiomyosarcoma with lymph node metastasis: A case report. World J Surg Oncol 14(1): 176, 2016. PMID: 27368929. DOI: 10.1186/s12957-0160936-Z

54 Ozturk H: Vena cava invasion by adrenal leiomyosarcoma. Rare Tumors 6(2): 5275, 2014. PMID: 25002946. DOI: 10.4081/ rt.2014.5275

55 Quildrian S, Califano I, Carrizo F, Daffinoti A and Calonico N: Primary adrenal leiomyosarcoma treated by laparoscopic adrenalectomy. Endocrinol Nutr 62(9): 472-473, 2015. PMID: 26215892. DOI: $10.1016 /$ j.endonu.2015.05.008

56 Thamboo TP, Liew LC and Raju GC: Adrenal leiomyosarcoma: A case report and literature review. Pathology 35(1): 47-49, 2003. PMID: 12701684. DOI: 10.1080/0031302021000062325

57 Zetler PJ, Filipenko JD, Bilbey JH and Schmidt N: Primary adrenal leiomyosarcoma in a man with acquired immunodeficiency syndrome (aids). Further evidence for an increase in smooth muscle tumors related to epstein-barr infection in aids. Arch Pathol Lab Med 119(12): 1164-1167, 1995. PMID: 7503667.

58 Zhou Y, Tang Y, Tang J, Deng F, Gong G and Dai Y: Primary adrenal leiomyosarcoma: A case report and review of literature. Int J Clin Exp Pathol 8(4): 4258-4263, 2015. PMID: 26097622.

59 Etten B, van Ijken MG, Mooi WJ, Oudkerk M and van Geel AN: Primary leiomyosarcoma of the adrenal gland. Sarcoma 5(2): 9599, 2001. PMID: 18521310. DOI: 10.1155/S1357714X01000184

60 Mencoboni M, Bergaglio M, Truini M and Varaldo M: Primary adrenal leiomyosarcoma: A case report and literature review. Clin Med Oncol 2, 2008. PMID: 21892297. DOI: 10.4137/ cmo.s627

61 Lokanatha D, Jacob L, Babu M, Lokesh K, Sai R, Rudresha A, Rajeev L, Saldanha S, Suma M and Usha A: Primary adrenal leiomyosarcoma: An extremely rare mesenchymal tumor. Indian J Med Paediatr Oncol 40(4): 559-562, 2019. DOI: 10.4103/ ijmpo.ijmpo_270_19

62 Rudin A, Torres-Mora J, Natt N and Richards M: Primary adrenal dedifferentiated leiomyosarcoma: A low grade leiomyosarcoma with a high-grade undifferentiated component: Case report. Clin Surg 1: 1193, 2016.

63 Nerli R, Ghagane S, Dixit NS, Hiremath MB and Deole S: Adrenal leiomyosarcoma in a young adult male. Int Cancer Confer J 9(1): 14-17, 2020. PMID: 31950011. DOI: 10.1007/ s13691-019-00387-1

64 Tzaida O, Papazian M, Kokkinos C, Provatas I, Markouizou A, Novkovic $\mathrm{N}$ and Nomikos I: Adrenal gland : An anatomical site of surprises: On occasion of a primary leiomyosarcoma. Ann Clin Exp Metabol 2(1): 1014, 2017.

65 Lujan MG and Hoang MP: Pleomorphic leiomyosarcoma of the adrenal gland. Arch Pathol Lab Med 127(1): 
e32-35, 2003. PMID: 12562292. DOI: 10.1043/00039985(2003)127<e32:PLOTA>2.0.CO;2

66 Deshmukh SD, Babanagare SV, Anand M, Pande DP and Yavalkar P: Primary adrenal leiomyosarcoma: A case report with immunohistochemical study and review of literature. J Cancer Res Ther 9(1): 114-116, 2013. PMID: 23575089. DOI: 10.4103/0973-1482.110394

67 Wang TS, Ocal IT, Salem RR, Elefteriades J and Sosa JA: Leiomyosarcoma of the adrenal vein: A novel approach to surgical resection. World J Surg Oncol 5: 109, 2007. PMID: 17910774. DOI: $10.1186 / 1477-7819-5-109$

68 Shao IH, Lee WC, Chen TD and Chiang YJ: Leiomyosarcoma of the adrenal vein. Chang Gung Med J 35(5): 428-431, 2012. PMID: 23127348. DOI: 10.4103/2319-4170.105475

69 Doppalapudi SK, Shah T, Fitzhugh VA and Bargman V: Primary adrenal leiomyosarcoma with inferior vena cava extension in a 70-year-old man. BMJ Case Rep CP 12(3): e227670, 2019. PMID: 30936336. DOI: 10.1136/bcr-2018-227670

70 Gulpinar MT, Yildirim A, Gucluer B, Atis RG, Canakci C, Gurbuz C and Caskurlu T: Primary leiomyosarcoma of the adrenal gland: A case report with immunohistochemical study and literature review. Case Rep Urol 2014: 489630, 2014. PMID: 24716083. DOI: 10.1155/2014/489630

71 Wei J, Sun A, Tao J, Wang C and Liu F: Primary adrenal leiomyosarcoma: Case report and review of the literature. Int $\mathrm{J}$ Surg Pathol 22(8): 722-726, 2014. PMID: 24619012. DOI: $10.1177 / 1066896914526777$

72 Pereira-Beceiro J, Rodriguez-Alonso A, Janeiro-Pais JM, Alvarez-Fernandez JC and Durana Tonder C: Adrenal pleomorphic leiomyosarcoma, an uncommon malignant suprarrenal tumour. Cir Esp 93(4): 256-258, 2015. PMID: 24703724. DOI: 10.1016/j.ciresp.2014.02.010

73 Sakellariou M, Dellaportas D, Grapsa E, Tzikanoulas M, Dellis A, Theodosopoulos $\mathrm{T}$ and Nastos C: Primary adrenal leiomyosarcoma: A case report. Mol Clin Oncol 12(4): 317-320, 2020. PMID: 32190312. DOI: 10.3892/mco.2020.1987
74 Shen WT, Sturgeon C and Duh QY: From incidentaloma to adrenocortical carcinoma: The surgical management of adrenal tumors. J Surg Oncol 89(3): 186-192, 2005. PMID: 15719374. DOI: $10.1002 /$ jso.20180

75 Grogan RH, Mitmaker EJ and Duh Q-Y: Changing paradigms in the treatment of malignant pheochromocytoma. Cancer Control 18(2): 104-112, 2011. PMID: 21451453. DOI: 10.1177/ 107327481101800205

76 Oda Y, Miyajima K, Kawaguchi K, Tamiya S, Oshiro Y, Hachitanda Y, Oya M, Iwamoto $\mathrm{Y}$ and Tsuneyoshi M: Pleomorphic leiomyosarcoma: Clinicopathologic and immunohistochemical study with special emphasis on its distinction from ordinary leiomyosarcoma and malignant fibrous histiocytoma. Am J Surg Pathol 25(8): 1030-1038, 2001. PMID: 11474287. DOI: 10.1097/00000478-200108000-00007

77 Weissferdt A, Phan A, Suster S and Moran CA: Adrenocortical carcinoma: A comprehensive immunohistochemical study of 40 cases. Appl Immunohisto Mol Morphol 22(1): 24-30, 2014. PMID: 23531850. DOI: 10.1097/PAI.0b013e31828a96cf

78 Tischler AS: Divergent differentiation in neuroendocrine tumors of the adrenal gland. Semin Diagn Pathol 17(2): 120-126, 2000. PMID: 10839612 .

79 Williams AR, Hammer GD and Else T: Transcutaneous biopsy of adrenocortical carcinoma is rarely helpful in diagnosis, potentially harmful, but does not affect patient outcome. Eur J Endocrinol 170(6): 829-835, 2014. PMID: 24836548. DOI: 10.1530/EJE-13-1033

Received June 7, 2020

Revised July 3, 2020

Accepted July 6, 2020 